\title{
Der messende Luchs
}

\section{Zwei verbreitete Fehler in der Galilei-Literatur}

\author{
Andreas Kleinert
}

The Measuring Lynx. Two Widespread Mistakes in the Galileo Literature

The article deals with two widespread mistakes concerning Galileo: A false quotation and a translation error. The quotation reads as follows: "Measure what is measurable, and make measurable what is not so." Although Galileo is quoted with these words in a large number of publications, the authenticity of the sentence is highly dubious because no one has ever provided a precise bibliographical reference for where to find it in Galileo's works. Galileo's alleged rule about measurement can be traced back to the works of two nineteenth century French scholars. This phrase was subsequently picked up by some internationally renowned scientists, who were responsible for its dissemination in German and English books and articles. The two English versions of the measurement quotation published by Hermann Weyl in the late forties of the last century strongly contributed to its worldwide diffusion. The sentence was even re-translated into German and French, and in recent scientific textbooks it is frequently used in order to characterize the methods of modern science. Notwithstanding its increasing popularity, referring to this expression as a quotation from Galileo is a striking example of academic sloppiness.

The translation error concerns the name of the Roman Academy of which Galileo was a member. Referring to its emblem, a lynx, Accademia dei lincei is often translated as "Academy of (the) Lynxes". But the Italian noun for Iynx is feminine (la lince), and the Italian translation of "Academy of the Lynxes" would be Accademia delle linci. The adjective linceo, however, means "Iynx-eyed" in the sense of "sharp-sighted", and therefore the correct translation of Accademia dei lincei is "Academy of the Lynx-Eyed".

Keywords: Galileo, measurement, false quotation, translation error, Academy of the Lynx-Eyed

Schlüsse/wörter: Galilei, Messen, falsches Zitat, Übersetzungsfehler, Accademia dei lincei

"Die Wissenschaft, sie ist und bleibt, / Was einer ab vom andern schreibt", dichtete Eugen Roth vor sechzig Jahren in der Einleitung seines Tierlebens. Das war nicht als fundierte Wissenschaftskritik gemeint, denn es geht weiter mit den Versen: „Und trotzdem ist, ganz unbestritten, / Sie immer weiter fortgeschritten." (Roth 1948: 3) Ärgerlich wird das unreflektierte Abschreiben jedoch immer dann, wenn das Abgeschriebene falsch ist und die Abschreiber aus purer Bequemlichkeit darauf verzichten, ihre Aussagen zu überprüfen. Zwei Beispiele aus der Wissenschaftsgeschichte sollen diese Abschreibepraxis verdeutlichen. 


\section{Messen, was messbar ist}

„Vor über 300 Jahren verkündete Galilei das Prinzip der modernen naturwissenschaftlichen Forschung: Man muß messen, was meßbar ist, und meßbar machen, was zunächst nicht meßbar ist." Dieses Zitat aus der Zeitung Die Welt vom 9. Januar 1988 war der Ausgangspunkt für eine Miszelle, die vor über zwanzig Jahren in den Berichten zur Wissenschaftsgeschichte erschienen ist (Kleinert 1988). Darin habe ich gezeigt, dass sich das Zitat, für das es keinen Beleg in den Werken Galileis gibt, bis 1941 zurückverfolgen lässt. Der Umstand, dass ich es nur in der deutschen Sekundärliteratur nachweisen konnte, sprach für die von Albrecht Fölsing geäußerte Vermutung, es sei wahrscheinlich die Erfindung eines deutschen Schriftstellers (Fölsing 1983: 476).

Mit Hilfe der Suchmöglichkeiten, die das Internet bietet, lässt sich diese Vermutung inzwischen ebenso widerlegen wie meine damalige Annahme, das Zitat komme nur in der deutschsprachigen Literatur vor. Schon 1847 schrieb Antoine-Augustin Cournot Galilei die erste der beiden im Zitat ausgedrückten Forderungen zu: Man müsse messen, was messbar ist. Bei ihm lesen wir:

La vraie physique a été fondée le jour où Galilée, rejetant des spéculations depuis si longtemps stériles, a conçu l'idée [...] de préciser la forme générale à donner aux expériences, en leur assignant pour objet immédiat la mesure de tout ce qui peut être mesurable dans les phénomènes naturels. (Cournot 1847: 375)

Abb. 1: Die älteste bisher nachgewiesene Stelle mit Galileis angeblicher Forderung, man müsse messen, was messbar ist (Cournot 1847: 375).

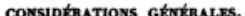

parlé) sont avec raison, à ce point de vue, réputées de pure curiosité, et ne cessent de paraltre telles que lorsque, pouvant faire avancer la théorie de la mesure des grandeurs, elles sont ou promettent d'être médiatement utiles à l'interprétation des phénomènes naturels.

Aussi, tandis que les philosophes, depuis Pythagore jusqu'à Kepler, avaient cherché vainement dans des idées d'ordre et d'harmonie, mystérieusement rattachées aux propriétés des nombres purs, l'explication des grands phénomènes cosmiques (1), la vraie physique a été fondée le jour où Galilée, rejetant des spéculations depuis si longtemps stériles, a conçu l'idée, non-seulement d'interroger la nature par l'expérience, comme Bacon le proposait de son cotté, mais de préciser la forme générale à donner aux expériences, en leur assignant pour objet immédiat la mesure de tout ce qui peut être mesurable dans les phénomènes naturels. $\mathrm{E} t$ pareille révolution a été faite en chimie un siècle et demi plus tard, lorsque Lavoisier s'est avisé de soumettre à la balance, c'est-à-dire à la mesure, des phénomènes dans lesquels on ne songeait généralement à étudier que ce par quoi ils se rattachent aux idées de combinaison et de forme. C'est cette méme direction que l'on poursuit dans l'étude de phénomènes bien plus compliqués encore, quand on táche de mesurer par la statistique tout ce qu'ils peuvent offrir de mesurable. 
Über zwanzig Jahre später hat dann Thomas Henri Martin die Forderung des Messbarmachens hinzugefügt: „Il [Galilée] déclare que dans tous ces phénomènes il faut mesurer tout ce qui est mesurable, et tâcher de rendre mesurable tout ce qui ne l'est pas directement." (Martin 1868: 289)

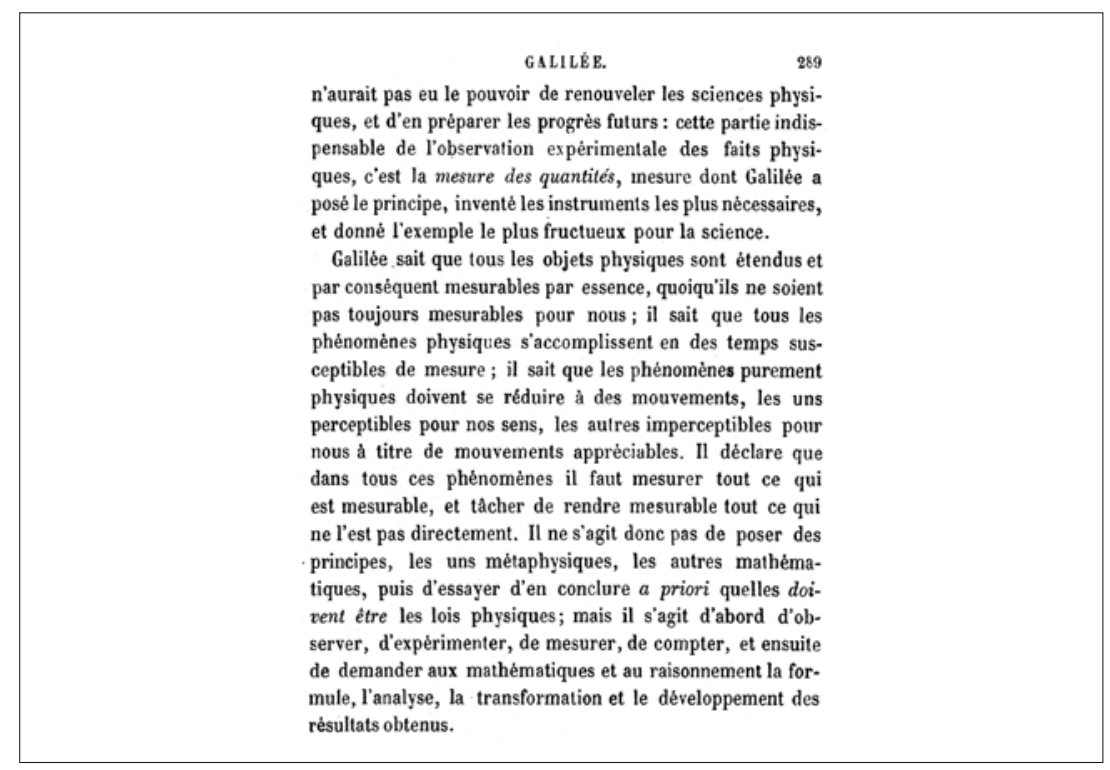

Abb. 2: Die älteste bisher nachgewiesene Stelle mit Galileis angeblicher Forderung, zu messen, was messbar ist, und zu versuchen, messbar zu machen, was nicht direkt messbar ist (Martin 1868: 289).

Vermutlich war der erste deutsche Verfasser, der sich im Zusammenhang mit dem Messen auf Galilei berief, der Philosoph Wilhelm Dilthey: „Alles messen, was messbar ist, und versuchen, messbar zu machen, was es noch nicht ist", sei Galileis Prinzip gewesen (Dilthey 1894: 45). Für zwei andere Galilei-Zitate auf derselben Seite gibt Dilthey genaue Quellenangaben in der Galilei-Ausgabe von Alberi (Galilei 1842-1856), für den Ausspruch über das Messen jedoch nicht. Zwanzig Jahre später wurde dieser Aufsatz im zweiten Band von Diltheys Gesammelten Schriften erneut abgedruckt, wodurch Galileis angebliche Forderung, alles zu messen oder messbar zu machen, noch leichter zugänglich wurde (Dilthey 1914: 259).

Wie Galileis Messprinzip von den eingangs genannten französischen Autoren zu Dilthey gelangt ist, lässt sich nicht eindeutig verfolgen. Es gibt jedoch ein handfestes Indiz, das vermuten lässt, wie Dilthey darauf gestoßen ist. Kurz nach dem Galilei-Spruch verweist er auf eine „ausgezeichnete Abhandlung von Natorp, Galilei als Philosoph“ (Dilthey 1894: 46). Natorp bringt in seinem von Dilthey zitierten Aufsatz zwar nicht das Galilei-Wort über das Messen, aber er erwähnt das oben genannte Buch von Henri Martin (1868), in dem sich beide Forderungen über das Messen zum ersten Mal nachweisen lassen: „Erst nach Vollendung dieser Skizze habe ich das Werk des Franzosen H. Martin über Galilei einsehen können." (Natorp 1882: 225). Martins 
Darstellung von Galileis Empirismus hält Natorp zwar für übertrieben, fügt aber lobend hinzu, dort werde „die Bedeutung der exacten Messung in der Methode Galilei's sehr treffend hervorgehoben“ (ebd.). Das könnte Dilthey veranlasst haben, selbst nachzulesen, was Martin über Galilei und das Messen geschrieben hat, und dabei wird er wohl auf das oben angeführte Zitat gestoßen sein, das er dann ohne Angabe der Quelle übernommen hat.

Dreizehn Jahre nach dem Erscheinen von Diltheys Gesammelten Schriften finden wir den Galilei-Spruch in identischer Formulierung in Hermann Weyls Beitrag zum Handbuch der Philosophie, was darauf schließen lässt, dass Weyl sich bei Dilthey bedient hat. Was dieser noch vorsichtig als "Galileis Prinzip“ bezeichnet hatte, wird bei Weyl endgültig zum Zitat: „Galilei spricht explizite als seinen Grundsatz aus: ,Alles messen, was meßbar ist, und versuchen, meßbar zu machen, was es noch nicht ist.' “ (Weyl 1927: 100)

1933 emigrierte Hermann Weyl in die USA. Dort führte er das angebliche Galilei-Zitat in die englischsprachige Literatur ein, und zwar in zwei Varianten, die bis heute etwa in gleicher Häufigkeit zitiert werden. Die erste lautet: „It was Galileo who said: ,Measure what is measurable, and make measurable what is not so." Weyl benutzte sie erstmals in einem Rundfunkvortrag, den er 1947 hielt und der noch im selben Jahr in einem populärwissenschaftlichen Sammelband veröffentlicht wurde (Weyl 1947: 141). 1959 wurde dieser Beitrag in einem anderen, ebenfalls für ein naturwissenschaftlich interessiertes Laienpublikum konzipierten Werk erneut abgedruckt, was zur weiteren Verbreitung des Zitats beitrug (Weyl 1959: 301).

Wer sich nicht auf populärwissenschaftliche Schriften berufen wollte, konnte auf die zweite von Weyl stammende englische Variante des Zitats zurückgreifen. Sie lautet: „Galileo enunciates the principle, ,to measure what is measurable and to try to render measurable what is not so as yet" ", und steht in der englischen Übersetzung von Weyls Handbuch-Beitrag von 1927, der 1949 unter dem Titel Philosophy of Mathematics and Natural Science in Princeton erschien und später mehrfach nachgedruckt wurde (Weyl 1949: 139). Seitdem wird Galilei unter Berufung auf Weyl von zahlreichen englisch publizierenden Autoren in einer der beiden Varianten zitiert (z.B. Rashid 2000: 224, Hellerman 2006: 539, Björk 2007: 23), wobei die erste, populärwissenschaftliche Variante überwiegt.

Im englischen Sprachraum hat sich das Zitat rapide verbreitet. Eine am 12. Januar 2009 durchgeführte Google-Suche nach „Measure what is measurable and make measurable what is not" und "Galileo" führte zu über 1.200 Treffern. Sogar in einem deutschen Lehrbuch über das Konsumverhalten verkündet inzwischen ein auf Englisch zitierter Galilei, was zu messen ist (Schirrmann 2005: 1). Auch nach Frankreich ist das Zitat auf dem Umweg über Deutschland und die USA wieder zurückgekehrt. Alain Carry zitiert Galilei unter Berufung auf Weyl mit den Worten „Nous devons mesurer ce 
qui est mesurable et rendre mesurable ce qui ne peut être mesuré“ (2003: 587). Auf Deutsch wird das Zitat weiterhin unverdrossen aus den Werken von Autoren abgeschrieben, die entweder keine Quelle angeben oder sich auf eine nicht dokumentierte Stelle eines anderen Verfassers berufen. Typische Beispiele aus neuerer Zeit sind Wilczek (2004: 243) und Haustein (2004: 159). Eine am 10. Januar 2009 angestellte Google-Suche nach „Messen, was messbar ist" und "Galilei“ ergab allein 680 Treffer.

Erwartungsgemäß blieben alle Anfragen nach der Quelle, die ich an zahlreiche Autoren richtete, die das Zitat benutzen (darunter viele Hochschullehrer), erfolglos. Als Rechtfertigung wurde von den Befragten unter anderem angeführt, dass diese Aussage doch „im Denken von Galilei liege“. So wird in einer eigenwilligen Interpretation der angeblichen Galileischen Devise durch einen nicht aufzuhaltenden Multiplikationsprozess ein Pseudozitat zitierbar gemacht, das nach den elementaren Regeln des wissenschaftlichen Arbeitens nicht zitierbar ist.

\section{Die Akademie der Luchse}

Galilei war seit 1611 Mitglied der 1603 von Federico Cesi gegründeten Accademia dei lincei, deren Name häufig mit "Akademie der Luchse“ übersetzt wird. Gleichartige Übersetzungen findet man auch in anderen Sprachen. Google lieferte am 11. Januar 2009 für „Akademie der Luchse“ 106 Treffer, 665 Treffer für „Academy of the Lynxes“, 653 Treffer für „Academy of Lynxes“ und 390 Treffer für "Académie des Lynx“. Beschränken wir uns auf die deutschsprachige wissenschaftshistorische Literatur, so begegnet uns die "Akademie der Luchse“ in den Gesamtdarstellungen der Geschichte der Physik von Poggendorff (1879: 209) und Heller (1882: 352) aus dem ausgehenden 19. Jahrhundert ebenso wie in zahlreichen Veröffentlichungen aus dem 20. und 21. Jahrhundert. Genannt seien die Galilei-Biographie von Fölsing (1983: 247) und als neuestes Beispiel die Studie über Galilei als Künstler von Bredekamp (2007: 149).

Ein Blick in ein beliebiges italienisches Lexikon lehrt, dass diese Übersetzung falsch ist. Der Luchs, das Wappentier der Accademia dei lincei, ist im Italienischen ein Femininum (la lince), was auch im darauf bezogenen Wahlspruch der Akademie („sagacius ista“) zum Ausdruck kommt (Maylender 1929: 437), in dem die Akademiker aus Rom den Anspruch erheben, noch scharfsichtiger als der Luchs zu sein. „Akademie der Luchse“ würde auf italienisch heißen Accademia delle linci. Der Name der Akademie enthält also nicht das Wort für Luchs, sondern das davon abgeleitete substantivierte Adjektiv linceo in der Bedeutung „scharfsichtig“ oder „luchsäugig“. Galilei war folglich kein Luchs, sondern ein scharfsichtiger Forscher, und die gelehrte Gesellschaft, der er angehörte, nannte sich „Akademie der Luchsäugigen“. 


\section{S T O R I A \\ E DIMOSTRAZIONI \\ INTORNO ALLE MACCHIE SOLARI \\ E LORO ACCIDENTI \\ COMPRESE IN TRE LETTERE SCRITTE \\ ALL'ILLVSTRISSIMO SIGNOR \\ MARCO VELSER I LINCEO \\ D V V M V I R O D'A V G V S T A CONSIGLIERO DI SVA MAESTA CESAREA \\ $D A L S I G N O R$ \\ GALILEO GALILEI LINCEO \\ Nobil Fiorentino, Filofofo,e Matematico Primario del Sereni/s. D. COSIMO II. GRAN DVCA DI TOSCANA. \\ Si aggiungono nel fine le Lettere, e Difquifizioni del finto Apelle.}

Abb. 3: Der Luchs im Wappen der Accademia dei lincei. Titelblatt von Galileis Schrift über die Sonnenflecken (Galilei 1613).

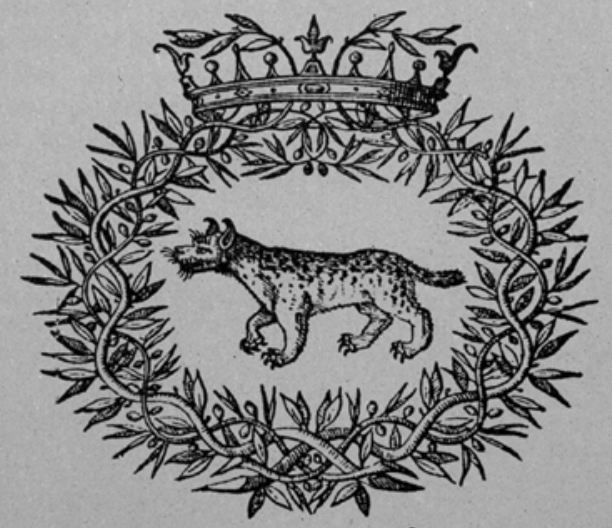

IN R OMA, Appreffo Giacomo Mafcardi. M DCXI II.

CON LICENZA DE' SVPERIORI.

Die korrekte englische Übersetzung ist „Academy of the Lynx-Eyed“. Obwohl einige Galilei-Biographen den Namen der Akademie korrekt übersetzt haben (etwa Krämer-Badoni 1983: 75), scheinen die Luchse in der GalileiLiteratur größere Überlebenschancen zu haben als in der freien Natur.

\section{„Zitiert nach"?}

Im Zeitalter von Google und Internet werden Artikel wie dieser wenig dazu beitragen, die Verbreitung erfundener Zitate und ähnlicher Fehler einzudäm- 
men. Wer macht sich schon die Mühe, Quellenstudien anzustellen, wenn er prägnante Formulierungen, deren Authentizität allein durch ihr häufiges Auftreten gesichert zu sein scheint, durch einfaches "copy and paste" in seine eigenen Texte übernehmen kann? Aber wenigstens Hochschullehrer und Herausgeber von Sammelbänden und Zeitschriften sollten durch eigenes Beispiel und durch Bestehen auf philologischer Strenge und Genauigkeit in Seminararbeiten und Dissertationen dafür sorgen, dass korrekt zitiert wird. Die Übernahme von Zitaten aus Sekundärquellen sollte nur dann zulässig sein, wenn dort die Primärquelle angegeben wird und das Original nicht oder nur mit großem Aufwand zugänglich ist. Alle anderen Formen von „zitiert nach" haben in der wissenschaftlichen Literatur nichts zu suchen.

\section{Literatur}

Björk, Anders, 2007. Chemometric and Signal Processing Methods for Real Time Monitoring and Modeling Using Acoustic Sensors. Applications in the Pulp and Paper industry. Diss. Stockholm [URL: www.diva-portal.org/diva/getDocument?urn_nbn_se_kth_diva-43832_fulltext.pdf].

Bredekamp, Horst, 2007. Galilei der Künstler. Der Mond, die Sonne, die Hand. Berlin: Akademie-Verlag.

Carry, Alain, 2003. Evolution de la dureé de scolarisation et effets sur la structure de formation de la population (France - dix-neuvième - vingtième siècles). Paedagogica Historica, 39, 583-598.

Cournot, Antoine-Augustin, 1847. De l'origine et des limites de la correspondance entre l'algèbre et la géométrie. Paris/Algier: Hachette.

Dilthey, Wilhelm, 1894. Die Autonomie des Denkens, der konstruktive Rationalismus und der pantheistische Monismus nach ihrem Zusammenhang im 17. Jahrhundert. Archiv für Geschichte der Philosophie, 8, 28-91.

Dilthey, Wilhelm, 1914. Die Autonomie des Denkens, der konstruktive Rationalismus und der pantheistische Monismus nach ihrem Zusammenhang im 17. Jahrhundert. In: Wilhelm Dilthey, Gesammelte Schriften, Bd. 2. Leipzig/Berlin: Teubner.

Fölsing, Albrecht, 1983. Galileo Galilei. Prozeß ohne Ende. München: Piper.

Galilei, Galileo, 1613. Istoria e dimostrazioni intorno alle macchie solari e loro accidenti. Rom: Mascardi.

Galilei, Galileo, 1842-1856. Le opere di Galileo Galilei. Prima edizione completa condotta sugli autentici manoscritti Palatini [da Eugenio Alberi]. Florenz: Società editrice Fiorentina.

Haustein, Heinz-Dieter, 2004. Quellen der Meßkunst. Berlin: de Gruyter.

Heller, August, 1882. Geschichte der Physik von Aristoteles bis auf die neueste Zeit, Bd. 1: Von Aristoteles bis Galilei. Stuttgart: Enke.

Hellerman, Leo, 2006. Representation of Living Forms. Biology and Philosophy, 21, 537-522.

Kleinert, Andreas, 1988. „Messen, was meßbar ist." Über ein angebliches Galilei-Zitat. Berichte zur Wissenschaftsgeschichte, 11, 253-255.

Krämer-Badoni, Rudolf, 1983. Galileo Galilei. München/Berlin: Herbig.

Martin, Thomas Henri, 1868. Galilée. Les droits de la science et la méthode des sciences physiques. Paris: Didier.

Maylender, Michele, 1929. Storia delle accademie d'Italia. Bd. 3. Bologna: Capelli.

Natorp, Paul, 1882. Galilei als Philosoph. Philosophische Monatshefte, 18, 193-229.

Poggendorff, Johann Christian, 1879. Geschichte der Physik. Leipzig: Barth.

Rashid, Salim, 2000. Economic Policy for Growth. Economic Development is Human Development. Boston u.a.: Kluwer. 
Roth, Eugen, 1948. Eugen Roths Tierleben. Mit Bilden von Julius Himpel. München: Hanser.

Schirrmann, Eric, 2005. Lokale Produktherkunft und Konsumentenverhalten. Der Einfluss der City-of-origin auf die Kaufentscheidung. Wiesbaden: Deutscher Universitäts-Verlag.

Weyl, Hermann, 1927. Philosophie der Mathematik und Naturwissenschaft. In: Alfred Baeumler und Manfred Schröter, Hg., Handbuch der Philosophie, Abteilung II: Natur/Geist/Gott. München/Berlin: Oldenbourg [o.S.].

Weyl, Hermann, 1947. Mathematics and the Laws of Nature. In: Warren Weaver, Hg., The Scientists Speak. New York: Boni \& Gaer, 140-143.

Weyl, Hermann, 1949. Philosophy of Mathematics and Natural Science. Princeton: Princeton University Press.

Weyl, Hermann, 1959. Mathematics and the Laws of Nature. In: Isabel S. Gordon und Sophie Sorkin, Hg., The Armchair Science Reader. New York: Simon and Schuster, 300-303.

Wilczek, Gerhard, 2004. Kleine Weltgeschichte der Philosophie. Norderstedt: Books on Demand.

Andreas Kleinert

Institut für Physik

Martin-Luther-Universität Halle-Wittenberg

Friedemann-Bach-Platz 6

D-06108 Halle/Saale

E-Mail: andreas.kleinert@physik.uni-halle.de 